\title{
Automatic Quality Electrogram Assessment Improves Reentrant Activity Identification in Atrial Fibrillation
}

\author{
Alejandro Costoya-Sánchez ${ }^{1}$, Andreu M Climent ${ }^{1,2}$, Ismael Hernández-Romero ${ }^{3}$, Alejandro Liberos ${ }^{1}$, \\ Francisco Fernández-Avilés ${ }^{2}$, Sanjiv M Narayan ${ }^{4}$, Felipe Atienza ${ }^{2}$, María S Guillem ${ }^{1}$, Miguel \\ Rodrigo ${ }^{1}$ \\ ${ }^{1}$ ITACA Institute, Universitat Politècnica de València, València, Spain \\ ${ }^{2}$ Hospital General Universitario Gregorio Marañón, Madrid Spain; ${ }^{3}$ Department of Signal Theory and \\ Communications, Rey Juan Carlos University, Spain; ${ }^{4}$ Cardiolovascular Department, Stanford \\ University, CA, USA
}

\begin{abstract}
Location of reentrant electrical activity responsible for driving atrial fibrillation $(A F)$ is key to ablative therapies. The aim of this work is to study the effect of the quality of the electrograms (EGMs) used for $3 D$ phase analysis on reentrant activity identification, as well as to develop an algorithm capable of automatically identifying lowquality signals.

EGMs signals from 259 episodes obtained from 29 AF patients were recorded using 64-electrode basket catheters. Low-quality EGMs were manually identified. Reentrant activity was identified in $3 D$ phase maps and provided an area under the ROC curve (AUC) of 0.69 when compared to a $2 D$ activation-based method. Reentries located in regions with poor-quality EGMs were then removed, increasing the AUC to 0.80. The EGM classification algorithm showed a similar performance both for low-quality EGM identification (sensitivity 0.91 and specificity 0.80) and for reentrant activity location with $3 D$ phase analysis (AUC 0.80).

Discard of reentrant activity identified in regions where EGMs showed low quality significantly improved the specificity of the $3 D$ phase analysis. Besides, EGMs classification according to their quality proved to be possible using time and spectral domain parameters.
\end{abstract}

\section{Introduction}

Catheter-based ablative therapy is one of the main approaches currently applied to restore sinus rhythm in patients suffering atrial fibrillation (AF), and specifically pulmonary veins isolation (PVI) has become the recommended procedure. Ablation therapies targeting atrial regions responsible for driving the $\mathrm{AF}$ process have proved to improve the clinical outcomes of these interventions [1]. These regions are commonly identified by the analysis of the electrogram (EGM) signals and driving regions can be identified as atrial sites containing repetitive reentrant activity.

EGM analysis for reentrant activity identification can be based on local activation detection, where activation instants for each EGM are reconstructed in order to identify circular wavefront patterns typical of electrical reentries [2]. On the other hand, phase-based methods rely on Hilbert-transformed maps to identify the reentrant activity as phase singularities. Both of those methods can be applied onto $2 \mathrm{D}$ or $3 \mathrm{D}$ maps, having the latter the advantage of taking the anatomy and the geometry of the catheter into account for the analysis.

However, atrial EGMs might be affected by noise from a variety of sources [3], such as bad contact of the electrode with the atrial wall or ventricular components. This noise might interfere with the activation- and phasebased analysis, causing the identification of false positives in non-driving regions of the atria. Still, manual identification of low-quality EGMs is very time consuming. Therefore, the goal of this work is to study the effect of the EGM recordings quality on reentrant activity detection, as well as to develop automatic classification algorithm able to identify low-quality signals.

\section{Methodology}

29 patients from the electrophysiology service of the Hospital GU Gregorio Marañón derived for AF ablation were included in this study. Intracardiac electrograms and atrial anatomy were obtained using 64-electrode intracavitary basket catheters. The study was approved by the ethics committee of the hospital, and all patients gave informed consent. Each EGM signal was then manually inspected and classified as a low- or high-quality signal. 


\subsection{Reentrant activity identification}

Atrial bipolar EGMs were filtered using a 2 to $20 \mathrm{~Hz}$ band-pass filter, followed by a $2 \mathrm{~Hz}$ bandwidth band-pass filter centered at the Highest Dominant Frequency (HDF) detected on each basket [4], obtained as the 95 percentile of the dominant frequencies (DFs) of the high-quality EGMs of the atrium. A 3D 1026-node mesh was created using the 3D distribution of the 64-poles basket catheter extracted from the navigation system in order to increase the model spatial resolution, and the recorded EGM signals were interpolated accordingly. Afterwards, rotor identification was achieved by applying the Hilbert transform to the interpolated filtered signals to obtain the corresponding phase map and identifying the phase singularities [4]. Only those consecutive phase singularities that completed at least one rotation were considered as rotors [3]. To determine the location of the rotors, the most frequented node by their phase singularities and their immediate neighbours were chosen.

Reentrant activity obtained with 3D-phase analysis was then compared to the reentries identified by a commercial mapping system (Ryhythm View, Topera, Abott) [1], based on signal activation analysis on 2D 8x8 maps. Reentries detected by the activation-based method were expanded to their neighbouring electrodes too in order to account for reentry movement. Both mapping methods were applied to the 144 recordings that lasted between 2 and 6 seconds, with an average of $4.1 \pm 0.9 \mathrm{~s}$ and with $12.6 \pm 9.6$ low-quality electrodes per episode.

The discard of reentrant activity according to EGMquality was implemented according to two different criteria. Using the strict criteria only those reentries with a low-quality EGM at their closest electrode were discarded. For the expanded criteria those reentries calculated near to low-quality electrodes were discarded, as well as the ones located in adjacent electrodes.

Sensitivity of the detection model was obtained for each episode as the number of $2 \mathrm{D}$ activation-detected reentries in which, in its expanded region, at least one of the electrodes was determined to harbor a reentry by the 3D phase-based method, compared to the total number of 2D activation-detected reentries on that episode. Specificity was calculated as the number of electrodes which did not belong to the extended regions of 2D activation-based reentries and were not identified by the 3D phase method, compared to the number of electrodes out of the extended regions of 2D activation-detected reentries. The ROC curves of the detection models were obtained choosing as a threshold the minimal temporal duration of these reentries, starting by 1 full rotation.

\subsection{Classification algorithm}

The quality-based EGM classification algorithm was trained using a random sample of signals from the database, consisting of 729 low-quality and the same number of high-quality EGMs. 30 parameters were calculated for each EGM from the time and spectral domain and used as descriptors for the algorithm, such as Power Spectral Density (PSD) under $3 \mathrm{~Hz}$ and the DF of the raw and filtered signal.

\section{Results}

\subsection{Reentrant activity identification by 2D-activation and 3D-phase mapping}

Reentries identified using the 3D-phase method (Figure 1C) were compared to those detected using the 2D-activation method (Figure 1B). In the whole cohort, the 3D-phase analysis without the discard of any reentrant activity provided a sensitivity of 0.93 , a specificity of 0.28 and an area under the ROC curve (AUC) of 0.59. Overall, the identification relied on the chosen temporal threshold, as reentries detected in the 3D-phase map were usually more intermittent, with an average duration of $279.50 \pm 185.7 \mathrm{~ms}$, while the reentries identified in the 2D-activation map lasted for tens of seconds.

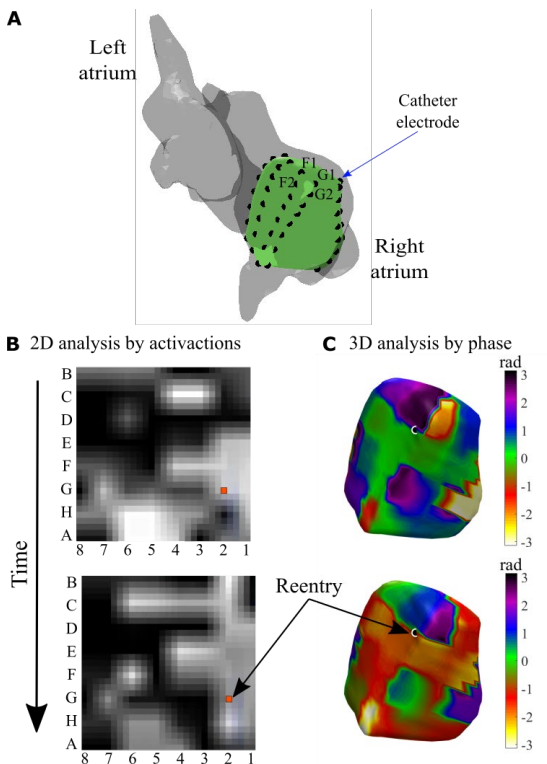

Figure 1. Example of 2D-activation and 3D-phase analysis. A. Basket catheter position within the right atria (electrode position marked with black spots). B. 2D activation sequence by $2 \mathrm{D}$-activation analysis, reentrant pattern marked with a red dot. C. 3D phase analysis sequence, reentrant pattern marked with a black dot.

\subsection{Effect of noisy EGM removal on reentrant activity identification}

Removal of reentries identified at atrial regions 
mapped with low-quality EGMs showed a significant improvement in the concordance between the 3D-phase analysis and the 2D-activation method. In Figure 2, an example of a reentry detected with high-quality EGMs coinciding with the 2D-activation method, and also a false reentry detected with low-quality EGMs can be appreciated, for the sample example of Figure 1.

A Rotor density with all recorded electrodes

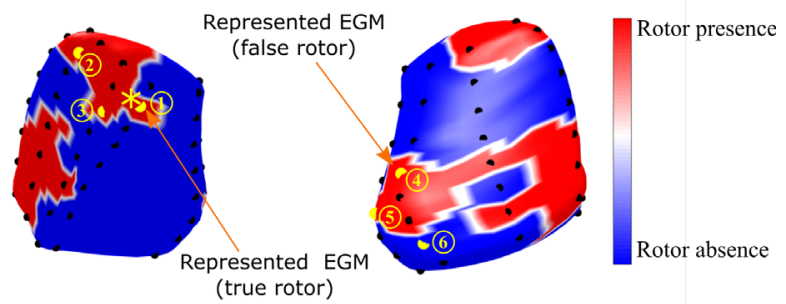

B EGMs around true rotor

C EGMs around false rotor

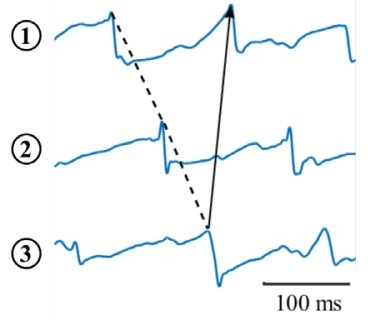

(4)

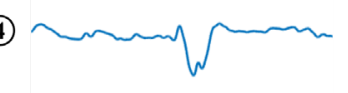

(5)

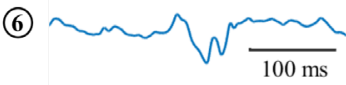

Figure 2. Example of true and false reentries based on EGM quality. A. Rotor density for all reentrant sources detected. Areas with reentrant activity are depicted in red. Rotor detected by 2D-method is marked with a yellow asterisk. B. EGMs marked in A around the 2D-method reentry. C. EGMs marked in A around a false reentry.

Discard of low-quality signals significantly increased the specificity of the 3D-phase method. In Figure 3, a qualitative example for the same case depicted in Figure 1 is showed, in which can be observed that many false positives were identified on regions with high density of low-quality EGMs (Figure 3B). Therefore, removal of reentries detected in atrial regions with low-quality EGMs improved the performance of the 3D phase-based algorithm. The ROC curves obtained from the reentrant activity comparison between the $2 \mathrm{D}$-activation and $3 \mathrm{D}$ phase methods for the whole cohort are depicted in Figure 4. The 3D-phase analysis without the removal of any reentrant activity provided an AUC of 0.69 , with a maximum sensitivity of 0.93 and a specificity of 0.28 , whereas when the strict removal criteria was applied, the AUC increased to 0.74 , without any decrease in sensitivity and with an improve in specificity to 0.42 . Moreover, for the reentrant activity identification with the expanded criteria the AUC value was 0.80 , with a maximum sensitivity of 0.92 and a specificity of 0.62 .

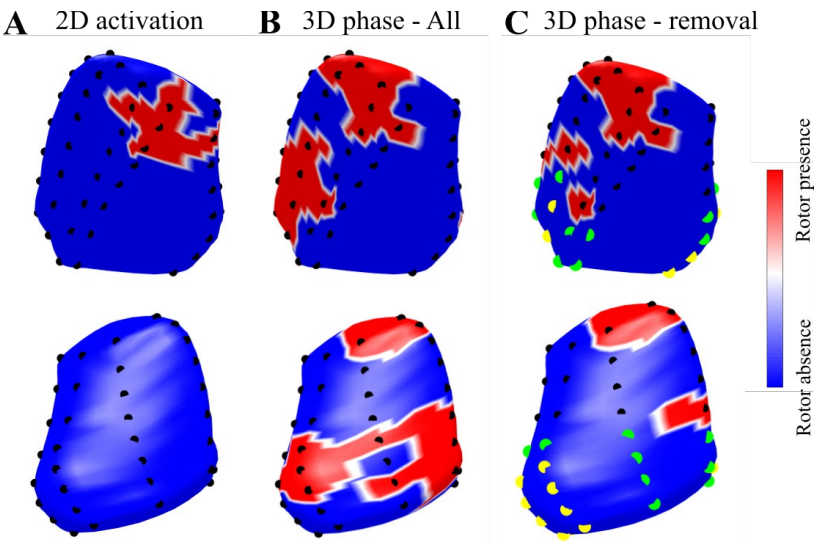

Figure 3. Example or reentrant activity histograms. Areas with reentrant activity are depicted in red. Reentries detected by A. 2D activation-based method; B. 3D phasebased method with all the EGMs; and C. 3D phase-based method removing low-quality EGMs. Low-quality EGMs are marked in yellow neighboring electrodes in green.
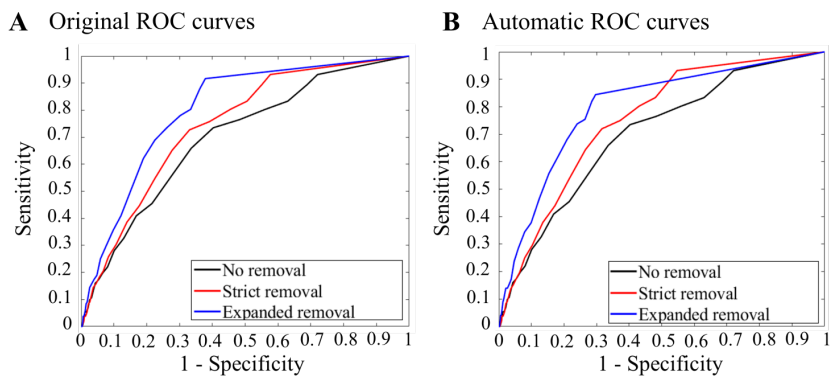

Figure 4. ROC curves of the 3D phase method: A. For manually classified EGMs; B. for automatically classified EGMs.

\subsection{Automatic detection of low-quality EGMs and reentrant activity identification}

Several classifying approaches were evaluated, from which tree decision with bagging and random forest had the best performance. This classification algorithm provided a sensitivity of 0.91 and a specificity of 0.85 in classifying low-quality signals, compared with manual annotation. The parameters used as descriptors that were estimated to be more significant to its performance were from the spectral domain, such as the spectral content under $3 \mathrm{~Hz}$ and the DFs of the raw and filtered signal.

The automatic EGM classification using the developed algorithm caused the misclassification of a significant number of high-quality EGMs, labeled as low-quality signals. These errors were more detrimental to the correct performance of the 3D-phase method than otherwise. Therefore, the classification algorithm was tuned to mark as low-quality only those EGMs with a probability of being so of $70 \%$ or higher according to the algorithm. With this setting, the EGM classification obtained a 
sensitivity of 0.92 and a specificity of 0.80 .

With this configuration, the 3D-phase analysis for reentries identification obtained a similar performance than with manually classified EGMs. When applied with the strict removal criteria, the AUC value increased to 0.75 , with a maximum sensitivity of 0.93 and a specificity of 0.45 . Moreover, when applying the expanded removal criteria the AUC was 0.80 , with a maximum sensitivity of 0.84 and a specificity of 0.70 .

\section{Discussion}

This work presents a novel study in which the relevance of the presence of low-quality signals in the reentrant activity detection was evaluated. In a cohort composed by $29 \mathrm{AF}$ patients, reentrant activity detection by a 3D phase-based method was compared to a commercial 2D activation-based algorithm, showing a high concordance with high sensitivity (0.93) but low specificity $(0.28, \mathrm{AUC}=0.69)$. However, reentrant activity removal in regions where low-quality EGMs have been recorded improved significantly its specificity $(0.62)$ while maintaining its sensitivity $(0.92, \quad \mathrm{AUC}=0.80)$, which demonstrates that EGM signal quality plays a significant role in the performance of the 3D phase-base methods. Moreover, an automatic algorithm based on tree decision with bagging and random forest was presented, capable of automatically classifying EGMs according to their quality based on time- and spectral-domain signal parameters. This algorithm showed an $\mathrm{AUC}=0.94$ for low-quality EGM identification, and 3D-phase analysis based on its automatic classification showed a similar performance than manual classification (sensitivity 0.84 , specificity $0.70, \mathrm{AUC}=0.80$ ).

This study, in line with others previously published $[5,6]$, has shown that both activation- and phase-based methods present similar results in reentrant activity detection. Moreover, the inclusion of 3D geometries allows to avoid false positives by taking into consideration the real 3D geometry of the mapped scenario, in cases of basket splines far away from each other or superimposed. However, phase-based methods rely heavily on signal quality, as noise induced by poor wall contact, ventricular artifacts or other reasons, can provoke false reentries identification. Therefore, inclusion of signal quality parameters in the phase-based algorithm allows to increase the concordance between activationand phase-based mapping systems.

Although ablation-guided therapies are not a major practice in the management of $\mathrm{AF}$, these have proved useful against traditional methods such as PVI [1]. This is the reason the most spread of them (RhythmView) was used as ground truth for this article. However, since it lacks from randomized clinical trials, this method cannot be considered as a gold standard.

\section{Conclusion}

Inclusion of signal quality parameters in the phase algorithm has proved to significantly improve the performance of the 3D phase-based method for reentrant activity detection. Moreover, classification of EGM according to their quality has been proved feasible with an automatic classification algorithm fed by time- and spectral-domain parameters of these signals. Application of reentrant activity removal criteria from atrial regions with poorly recorded EGMs results into an improvement of the identification of AF-driving sources, and therefore, a possible improvement of the ablation-guided procedures. Besides, automatic EGMs quality assessment may be used to indicate clinicians which regions of the atrium should be rerecorded.

\section{References}

[1] Narayan SM et al., "Direct or Coincidental Elimination of Stable Rotors may Explain Successful Atrial Fibrillation Ablation: on-Treatment Analysis of the CONFIRM Trial," $J$ Am Coll Cardiol, vol. 62, no. 2, pp. 138-147. Jul, 2013.

[2] Zaman JA et al., "Identification and Characterization of Sites Where Persistent Atrial Fibrillation Is Terminated by Localized Ablation," Circ Arrhythm Electrophysiol, vol. 11, no. 1, pp. e005258. Jan, 2018.

[3] Kuklik P et al., "Identification of Rotors during Human Atrial Fibrillation Using Contact Mapping and Phase Singularity Detection: Technical Considerations," IEEE Trans Biomed Eng, vol. 64, no. 2, pp. 310-318. Feb, 2017.

[4] Rodrigo $\mathrm{M}$ et al., "Highest Dominant Frequency and Rotor Positions are Robust Markers of Driver Location during Noninvasive Mapping of Atrial Fibrillation: A Computational Study," Heart Rhythm, vol. 14, no. 8, pp. 1224-1233. Aug, 2017.

[5] Rodrigo $\mathrm{M}$ et al., "Technical Considerations on Phase Mapping for Identification of Atrial Reentrant Activity in Direct- and Inverse-Computed Electrograms," Circ Arrhythm Electrophysiol, vol. 10, no. 9, pp. e005008. Sep, 2017.

[6] Vidmar D et al., "Characterizing Electrogram Signal Fidelity and the Effects of Signal Contamination on Mapping Human Persistent Atrial Fibrillation,”. Front Physiol, vol. 9, pp. 1232. Sep, 2018.

\section{Acknowledgements}

This work was supported by the Instituto de Salud Carlos III FEDER (DTS16/00160; PI16/01123; PI17/01059; PI17/01106), the EIT-Health 19600 AFFINE and the Valencian Regional Government (AICO2018).

Address for correspondence:

Miguel Rodrigo

ITACA Institute, Universitat Politècnica de València. mirodbor@teleco.upv.es 